\title{
Control interno para mejorar la gestión patrimonial del instituto enrique lopez
}

albujar

\section{Internal control to improve the institute's asset management enrique lopez albujar institute}

\author{
Claudia De la Cruz, ${ }^{1}$ Lourdes Casas, Wilmer Vidaurre, ${ }^{3}$ Max Urbina, Carmen Rosas ${ }^{5}$
}

\section{RESUMEN}

El propósito principal de la indagación es proponer actividades de control interno que mejoren la gestión de bienes en el Instituto Enrique Lopez Albujar, Ferreñafe. La investigación se enmarcó en el tipo propositivo, con diseño no experimental. Como población y muestra se consideran a los empleados de la entidad, como instrumento para recolectar la información se utilizó el análisis documental en los documentos del área de abastecimiento y un cuestionario con escala de Likert. El resultado muestra que es necesario diseñar actividades de control interno para mejorar gestión de los bienes de la institución en el área patrimonial debido a qué sus resultados de evaluación son deficientes y se agudizan por la emergencia sanitaria por COVID 19. Se concluye que es impostergable aplicar las actividades de control interno.

Palabras Clave: Control Interno, gestión, Gestión de Bienes

\section{ABSTRACT}

The main purpose of the inquiry is to propose internal control activities that improve asset management at the Enrique Lopez Albujar Institute, Ferreñafe. The research was framed in the propositional type, with a non-experimental design. As population and sample, the employees of the entity are considered, as an instrument to collect the information, the documentary analysis was used in the documents of the supply area and a questionnaire with a Likert scale. The result shows that it is necessary to design internal control activities to improve the management of the institution's assets in the heritage area due to the fact that its evaluation results are deficient and are exacerbated by the health emergency caused by COVID 19. It is concluded that it is urgent to apply internal control activities.

Key Words: Internal Control, management, Asset Management

1. Estudiante en Contabilidad, Universidad Señor de Sipán S.A.C., Pimentel - Chiclayo, Perú, rsancheznalmiyo@crece.uss.edu.pe 2. Estudiante en Contabilidad, Universidad Señor de Sipán S.A.C., Pimentel - Chiclayo, Perú, csantistebanshi@crece.uss.edu.pe

3. Doctor en Educación, Maestro en Docencia y Gestión Universitaria, Escuela de Contabilidad, Facultad de Ciencias Empresariales, Universidad Señor de Sipán S.A.C., Pimentel - Chiclayo, Perú, vidaurrewil@crece.uss.edu.pe

4. Vicerrector de Investigación, Innovación y Emprendimiento Universidad Alas Peruanas, Contador Público y Licenciado en Administración egresado de la Universidad Nacional de Trujillo, Maestro en Ciencias Económicas con mención en Finanzas egresado de la Escuela de Posgrado de la Universidad Nacional de Trujillo, Vicerrector de Investigación Universidad Alas Peruanas, urbina10@hotmail.com

5. Doctora en Educación, Magíster en Docencia Universitaria, Maestra en Ciencias Económicas con mención en Tributación Contadora Publica, Licenciada en Administración, Docente Ordinaria de la Universidad Señor de Sipán, categoría principal, celvirarp@crece.uss.edu.pe 


\section{INTRODUCCIÓN}

Transparency internacional (2018). Afirma que los presupuestos de la mayoría de los estados del mundo tienen un porcentaje representativo con respecto al gasto de contratación o adquisición de bienes o servicios, esto es el $70 \%$ del total del mismo. Para la adquisición de lo mencionado y autorización del gasto se pasa por un proceso exhaustivo, sin embargo, en estos procedimientos se presentan indicios de corrupción, perjudicando seriamente a los ciudadanos, debido a que los fondos destinados para el desarrollo del país pasan a bolsillos de algunos funcionarios. Estos problemas se han presentado con mayor frecuencia en países de América Latina, con grandes escándalos de corrupción que desacredita la imagen de las instituciones públicas. Para Cuevas (2018) la mayoría de países tiene problemas en el control interno de las instituciones públicas, el cual es el encargado de estudiar la información financiera fraudulenta en las mismas y que no se aplican a tiempo para prevenir problemas de corrupción a grandes escalas.

OSCE (2019) El Perú, cuenta con un Organismo Supervisor de las Contrataciones del Estado (OSCE), afecto al MEF, tiene por objetivo principal fiscalizar cada uno de los procesos de las contrataciones todo ello mediante un plan respectivo, ello acredita la transparencia y evita asuntos de corrupción. A través de la promoción de buenas prácticas en la ejecución de cada uno de los procesos. A su vez, también supervisa la contraloría del estado, organismo brinda apoyo y asesoramiento respecto al gasto público la Contraloría general del estado (2014).

En este sentido Fonseca (2011), conceptualiza como control interno al "proceso realizado por medio de un comité dirigente, oficinista, funcionario y otros integrantes de la entidad, cuya finalidad es la proporción de la solidez y comunicación comprensible visualizando si se cumplen con el propósito establecido". El control interno como sistema nos garantiza la prevención de irregularidades, riesgos, y además los actos de corrupción que se pueden presentar en una entidad pública, todo ello gracias a la aplicación de cada uno de los elementos organizacionales como la planeación, el mismo control de gestión, evaluación, organización, así como los sistemas de información y comunicación. Finalmente, el control intrínseco dicho en otras palabras se conceptualiza como un proceso multidireccional, pues cada uno de sus componentes tiene influencia sobre los demás, ya que todos estos son parte de un sistema integrado.

En la gestión patrimonial, se denomina gestión al monitoreo y ejecución de mecanismos con la finalidad de concretizar y continuar objetivos de una organización. Esto por consiguiente requiere de compromiso tanto de la institución como de todos los colaboradores que se involucren con principios y valores. Desde otra perspectiva se entiende que la administración de toda entidad se aplican técnicas de gestión para desarrollar acciones y lograr objetivos.

Gestión (2018), en una de sus publicaciones nos indica que un conjunto conformado por 185 municipios provinciales, representan el 93\% de las 196 que existen en Perú, cuentan con la oficina de Control Institucional (OCI) con el fin de reforzar el control estatal, inspeccionar el uso adecuado de los fondos públicos, cautelando la ley y eficacia en estas instituciones. Además, señaló que se viene fortaleciendo estos organismos con equipos de profesionales capacitados, el cual estará integrado por ingenieros, auditores, abogados. Snack mencionó que antes del proceso de expansión del control nacional y asimilación del OCI, el treinta por ciento de los municipios provinciales carecían de estos organismos, además las dos terceras partes restantes que, si lo venían implementado, el $50 \%$ de ellas; los trabajadores eran de la misma institución y no de la Contraloría. En vista de esto, estamos seguros que esto no prueba la autonomía, independencia y eficiencia de verificar en la gerencia de los fondos públicos. 
En unos de sus artículos, Colima (2019), señaló que el gobierno del estado ha restablecido la eficacia de la gerencia patrimonial, a través de la secretaría administrativa y el departamento de gestión pública en donde han realizado capacitaciones acerca del nuevo sistema de control patrimonial, convirtiéndose ello en una herramienta a actualizar de alguna manera. En el caso de activos de alta eficiencia, esta aplicación ayudará al gobierno a controlar los activos en uso porque le permitirá conocer las condiciones específicas de los activos, cuántas veces se han utilizado y el historial de siniestros

Gómez (2019), el cual realizó una investigación sobre la gestión de inventarios, siendo su objetivo elaborar estrategias para su mejoramiento. La metodología utilizada fue descriptivo, analítico y explicativo. La muestra estuvo conformada por diez integrantes de la entidad. La técnica que aplicó es la observación, encuesta y entrevista. Finalmente, vemos la importancia que se tiene implementar procesos para un eficiente control. Además, se concluye que la evaluación de gestión patrimonial del instituto, presenta falencias al momento de la regulación y generación de PECOSAS, puesto que no se viene cumplimiento con la normativa estipulada.

En la actualidad la institución presenta una deficiencia en sus actividades de control de bienes patrimoniales por el desconocimiento del tema por parte del personal jerárquicos, administrativos y docentes, también por la no implementación de una oficina de control interno en el instituto, lo cual conlleva a una dificultad en la unidad de abastecimiento por la inexistencia de un control sobre las actividades.

En varias ocasiones en el área de almacén no se da cumplimiento a cabalidad con el protocolo establecido por la institución al momento de realizar sus requerimientos, pues los usuarios no cumplen con los plazos establecidos. La problemática se origina porque se entrega el material o bienes solicitados, pero no se genera la PECOSA de manera oportuna, generando ello inconvenientes como desconocimiento de situación actual de los mismos, por el cual cuando se realiza el cruce de información no permite tener datos reales en los estados financieros, adicional a ello no se realizan las compras de los materiales de manera oportuna.

Cruz (2019), a través de su informe de estudio sobre el control interno del inventario en un sistema educativo, incluyó diseñar un sistema para este con la finalidad de su mejoramiento, el modelo de estudio fue descriptivo, el cual tuvo como población y muestra a todos los empleados contables-administrativos del ente, que son aquellos que están directamente relacionados con la gestión de inventarios, se aplicaron técnicas como las entrevistas, encuesta, y análisis documental para recolectar datos, el autor concluyó de que el trabajador contable no conoce el proceso que debe ejecutarse al ingresar nueva mercancía, esto acarrea a determinar decisiones perjudicando así la escasez de la entidad. Además de ello existe falta de registro minucioso en todos los periodos de la mercancía y falta de una orientación que indique como se va llevar, en que forma y realiza el cálculo de inventario para efectuar la operación de registro de inventario.

Balla y López (2018), mediante el estudio referente al control interno en la dirección administrativa, tuvo por objetivo describir $y$ determinar el dominio que este posee en el mejoramiento de la gerencia administrativa en las entidades ecuatorianas, el tipo de análisis fue documental al cual se revisó información más importante extraída de fuentes diferentes, se empleó técnicas de interpretación textual. Se concluyó que el control interno es el instrumento el cual se debe implementar en todas las entidades sea se encuentren en uno o en otro grupo económico, esto con el fin de ver su eficaz y eficiente información financiera, las operaciones, ordenamiento de normas y leyes. Dentro del control intrínseco es de suma importancia contar con una fuerza laboral calificada que cumpla con los objetivos establecidos evaluando, cautelando, así como dar seguimiento para obtener un 
control interno eficiente.

drovo y Córdova (2018), a través de su informe referentealcontrolinterno delos inventarios, tuvo por objetivo identificar el nivel de repercusión por no implementar un control interno así mismo el diseño de un manual para los inventarios, utilizó la indagación documental, su población y muestra estuvo conformada por todo el equipo administrativo de la hacienda, para recolección de datos se aplicaron las encuestas, el cual llegó a la conclusión de que se formularon los debidos procesos y políticas para los trabajadores, en ella se detalla las responsabilidades que tienen que cumplir en desempeñar su labor. Adicional, se determinó que los documentos si se encuentran acorde a la lista de control de ingreso, egresos e inventario para que estos sean utilizados y anotar la información minuciosa la cual servirá de apoyo para llevar una buena gestión en el ente.

Peralta y Villavicencio (2018), en la investigación del control interno, tuvo por finalidad general la elaboración de un plan de mejora para el control intrínseco, con la finalidad del aumento de la producción, asimismo el cumplimiento de objetivos de manera eficaz, utilizó el modelo de investigación descriptivo, con un enfoque mixto, para obtener datos aplicó técnicas tales como, análisis documental, entrevistas, y encuestas, se concluyó que el control intrínseco es un instrumento de apoyo muy importante, pues permite lograr los objetivos de un ente, ya que asegura que las actividades se cumplan de manera eficiente y eficaz. Y la evaluación del control intrínseco nos permite la identificación de falencias y zonas críticas para así implementar revisiones y mejoras en el control.

Estupiñan (2012) nos menciona que el control "es la transformación de determinar lo que se ejecuta, evalúa, por consiguiente, aplicar una medida correctiva cuando sea necesario, para que la ejecución proceda según lo planeado. En tanto Kirschenbaum (2004) indica que el control interno es el apoyo de actividades y operaciones de la organización, es decir, produce, distribuye, financia, administra y entres otras.
Es el desarrollo que lleva a cabo la dirección, administración y equipos de la empresa con base al desarrollar políticas para la organización, con el fin de identificar suceso potencial que afecten la entidad.

Mantilla (2005), Es una herramienta eficaz, no un proyecto para brindar algún tipo de supervisión policial o autoritaria. El método de control interno es no perjudicar el enlace entre la empresa, usuarios y compradores un alto grado de dignidad humana. Empleador a empleado. Menciona también Lozano (2019), Según el autor para poseer un control interno eficiente es necesario la implementación de ciertas herramientas de administración las cuáles ayudarán a controlar sus actividades a desarrollar ya que esto permitirá la verificación del cumplimiento de objetivos, así mismo obtener un diagnóstico a corto plazo de todas las funciones que se ejecutan en cada área.

Cabrera (2018), mediante su estudio referente a la repercusión que tiene el control interno sobre los bienes, siendo su objetivo principal la demostración del suceso del control intrínseco patrimonial en la gerencia de estos, para el estudio se determinó como su población y muestra el área de "Administración y Finanzas" el cual 18 trabajadores lo conforman y el acervo documental patrimonial. Para recolectar data, la entrevista se aplicó. Según, Jiménez (2010), el control en el patrimonio consiste en realizar coordinaciones para elaborar el inventariado físico del patrimonio mobiliario del ente, con la finalidad del otorgamiento de información necesaria en los diferentes organismos del Estado. Ramón (2019), a través de su informe referente al control de activos fijos, como objetivo general se propuso determinar criterios técnicos, para así tener una administración eficiente y mayor control de estos, llegó a la conclusión que la institución de estudio no tiene una unidad que controle los bienes, a través de procedimientos y mecanismos en el cual permita la ubicación y localización oportuna de los bienes. Así también Niño (2017), mediante su investigación referente al establecimiento de un conjunto de acciones 
con miras al mejoramiento de la administración de los inventarios, tuvo como objetivo sugerir que la entidad implemente un control intrínseco con el fin del mejoramiento de su administración

\section{MÉTODO Y MATERIALES}

La investigación fue de tipo descriptivo propositivo con diseño no experimental porque se demostró la hipótesis de trabajo mediante análisis y con el juicio de expertos se validaron este informe lo que permitirá mejorar en el control intrínseco, la población del estudio estuvo conformada por los trabajadores, y los documentos del área de abastecimiento.

La técnica empleada fue el cuestionario el cual se ejecutó a través de un formulario con preguntas en escala de Likert, es dirigido a los empleados que llevan a cabo el control interno, y el análisis documental se revisó y selecciono la averiguación importante de la entidad, ello mediante un análisis de PECOSAS del año 2020, con el fin de manifestar su contenido sin ambigüedades la que después será evaluada por las investigadoras.

Los instrumentos permiten compendiar la información sobresaliente a la realidad problemática de analizar, evaluar e interpretar. Mencionamos que los instrumentos aplicados poseen un alto grado de validez, fiabilidad y objetividad de asegurar la verdad de la averiguación y alcance del estudio.

\section{RESULTADOS}

Tabla N ${ }^{\circ}$ 1. Control Interno Actual

\begin{tabular}{lcc}
\hline \multicolumn{1}{c}{ Categoría } & $\mathbf{N}$ & $\mathbf{\%}$ \\
\hline Deficiente & 9 & 19.15 \\
Regular & 22 & 46.81 \\
Bueno & 16 & 34.04 \\
Eficiente & 0 & 0.00 \\
Total & 47 & 100.00 \\
\hline
\end{tabular}

Fuente. Elaboración propia

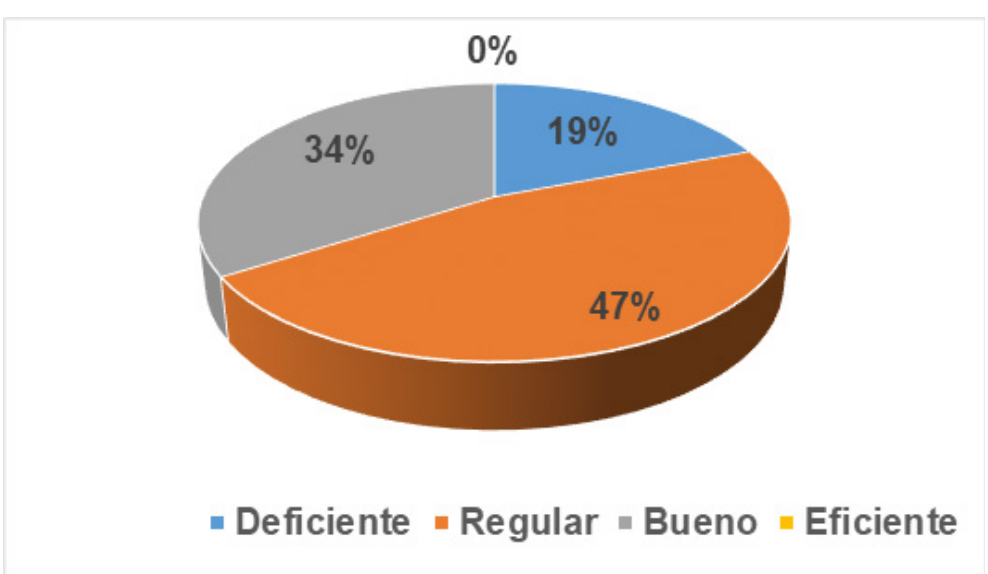

Figura 1 Control Interno Actual Interpretación:

De acuerdo a los resultados en general, el 46.81\% nos indica que el control interno de la institución se encuentra en términos regulares, es decir nos dicen que aún hay presencia de ciertas falencias en los procesos estipulados, así mismo, el 34.04\% dijo bueno y el otro 19.15\% se mostró deficiente. 
Tabla 2. Actividades de control Interno.

\begin{tabular}{|c|c|c|c|c|}
\hline $\mathbf{N}^{\circ}$ & Actividades & Responsable & Tiempo & Beneficiario \\
\hline 1 & $\begin{array}{l}\text { Establecer estrategias para } \\
\text { el cumplimiento de las } \\
\text { normas del instituto. }\end{array}$ & $\begin{array}{l}\text { Dirección / } \\
\text { Administraci } \\
\text { ón }\end{array}$ & Trimestral & Entidad \\
\hline 2 & $\begin{array}{l}\text { Establecer el } \\
\text { de lenocimiento } \\
\text { organizacional. }\end{array}$ & $\begin{array}{c}\text { Dirección / } \\
\text { Administraci } \\
\text { ón }\end{array}$ & Mensual & Entidad \\
\hline 3 & $\begin{array}{l}\text { Establecer objetivos } \\
\text { medibles para la } \\
\text { organización por parte del } \\
\text { componente humano. }\end{array}$ & $\begin{array}{l}\text { Organización } \\
\text { completa }\end{array}$ & Permanente & Entidad \\
\hline 4 & $\begin{array}{l}\text { Establecer políticas para la } \\
\text { gestión de riesgos } \\
\text { Utilizar medios de }\end{array}$ & $\begin{array}{l}\text { Dirección / } \\
\text { Administración }\end{array}$ & Permanente & Entidad \\
\hline 5 & $\begin{array}{lr}\text { comunicación } & \text { para } \\
\text { comunicar } & \text { información } \\
\text { relevante. } & \end{array}$ & $\begin{array}{c}\text { Dirección / } \\
\text { Administración }\end{array}$ & Permanente & Entidad \\
\hline 6 & $\begin{array}{l}\text { Inspeccionar de forma } \\
\text { sistema de gestión y control. }\end{array}$ & $\begin{array}{c}\text { Dirección / } \\
\text { Administración }\end{array}$ & Permanente & Entidad \\
\hline
\end{tabular}

Fuente. Elaboración propia

Interpretación:

Esta lista se han elaborado actividades con las que se pretende mejorar el control interno de bienes patrimoniales, con la finalidad de disminuir el desconocimiento por partes de los trabajadores. Las actividades se han planteado para progresar la gestión y asegurar el logro de las metas institucional.

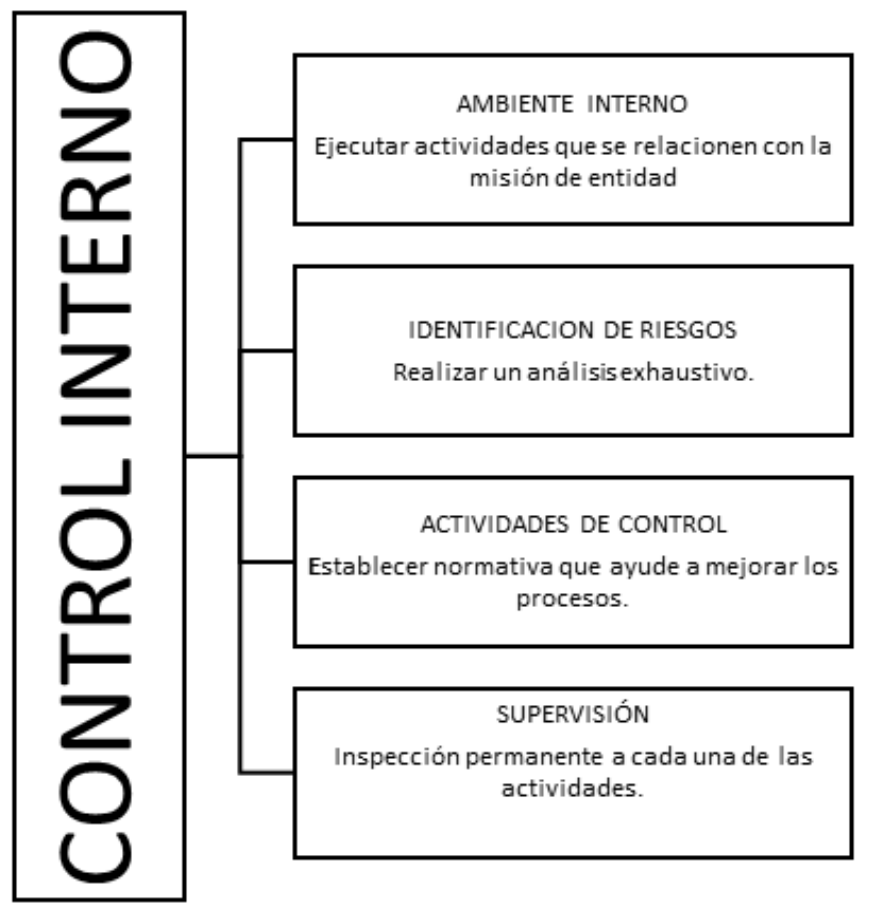

Fuente. Elaboración propia 


\section{Interpretación:}

Este esquema se ha elaborado actividades, con la finalidad de dar conocer a los trabajadores. Las actividades se han propuesto para el mejoramiento de la gestión de los procesos .

\section{DISCUSIÓN}

El propósito de la indagación es proponer actividades de control interno para mejorar la gestión de bienes patrimoniales, como resultados dichas actividades se han estipulado de acuerdo al ambiente de la institución, tal como: el establecimiento de estrategias para el cumplimiento oportuno de la normativa, brindar un conocimiento de la estructura organizacional de la institución, establecer objetivos medibles, así como también el establecer políticas de riesgos y la inspección del sistema de gestión y control.

Como conclusión se tiene que mediante la aplicación de estas actividades se logra mejorar el control interno en la dirección del patrimonio de la institución.

Esta opinión tiene coherencia con el concepto de

Fonseca (2015), pues nos comenta que el control interno es ejecutado por administrativos cuya finalidad es proporcionar solidez y comunicación comprensible en uno de los procesos, con el fin de cumplir con el propósito planteado.

En lo concerniente al objetivo específico: Evaluar la gestión patrimonial de la Institución.

Se observó la existencia de ciertas falencias en la generación de PECOSAS, pues se solicitan bienes, y dan salidas del almacén sin la generación oportuna de este comprobante, y donde después que realizan la regulación de la misma no se cumple con la normativa estipulada. El análisis de las PECOSAS vemos que no se cumple con la normativa pues hay salidas de bienes sin la generación de la misma. El resultado confirma que la teoría de Colima (2019) se refiere a la importancia que tiene que mejorar la gestión patrimonial, brinda una información acerca de los activos de la entidad con el fin tener un mayor control. Además, coinciden Gómez (2019), quien concluyo la importancia que se tiene de implementar procesos para un eficiente control.

En lo referente al objetivo específico: Describir el control interno actual, Aplicó una encuesta compuesta con 17 preguntas. En la encuesta aplicada llegamos a los resultados de que el control interno se viene realizando de forma regular, puesto que el $47 \%$ nos ha brindado esa información, lo cual nos da entender de la presencia de falencias en los procesos. A decir Guzmán (2013), menciona que el control patrimonial juega un rol relevante pues se muestra como una herramienta de gestión con el fin de mejorar la eficaz dirección de los bienes.

Coinciden también García y Nazareno (2018) concluyo la importancia de un eficiente control interno, este influye formular en la gestión de bienes, con la finalidad de menguar riesgos y así evaluarlos de forma oportuna.

Finalmente correspondiendo al objetivo específico: Diseñar actividades de control para restablecer la gestión de bienes se pretende mejorar eficiencia en la gestión de recursos de la organización. Las actividades han sido diseñadas de acuerdo a las teorías. Se analizó en base a Gonzales y Huamán (2018) su estudio al referente al planteamiento de un sistema que controle el patrimonio con el fin de reducir los faltantes de un municipio. Se aplicó encuestas, observación y entrevistas. Hemos concluido de la importancia del cumplimiento de todos los procesos y procedimientos. Se relaciona con el estudio de Sánchez (2017), sobre el control interno patrimonial para el mejoramiento en la distribución logística en un ente de Ferreñafe, siendo su objetivo indicar que el control interno brindará mejorías a la gestión patrimonial y posee ocurrencias en la entidad 


\section{CONCLUSIONES}

La propuesta en diseñar de actividades de control interno si lograra mejorar la gestión de bienes, que permitan desarrollar todas las actividades. dentro de los resultados se han estipulado de acuerdo al ambiente de la institución, en la cuales tenemos: el establecimiento de estrategias para cumplir la regla, brindar conocimientos de la empresa, implementar objetivos, establecer políticas y la inspección del sistema, va ayudar a controlar los recursos del esta y demostrando una información transparente y oportuna.

$\mathrm{Al}$ evaluar la gestión de bienes de la institución encontramos que tiene muchas falencias en os comprobantes de salida no cumplen con las normas

Se observa los resultados del control interno se vienen realizando de forma regular, puesto que el $47 \%$ nos dan a conocer, en el cual entendemos de la presencia de errores en los procesos en el cual afecta en el objetivo de la entidad.

Al plantear acción de control interno se logrará mejorar la eficaz de la gestión de bienes en el instituto, las actividades han sido diseñadas de acuerdo a las doctrinas, responder a las actividades mediante acciones concretas, comunicar e informar.

\section{REFERENCIAS}

Balla, I. y López, k. (2018). El Control Interno en la Gestión Administrativa de las Empresas del Ecuador (Trabajo de Titulación de Grado, Universidad Estatal de Milagro - Ecuador). Repositorio de Universidad Estatal de Milagro. http://repositorio.unemi. edu.ec/bitstream/123456789/4022/3/EL\%20 CONTROL\%20INTERNO\%20EN\%20LA\%20 GESTION\%20ADMINISTRATIVA\%20DE\%20 LAS\%20EMPRESAS\%20DEL\%20ECUADOR. pdf
Colima, D. (20 de febrero 2019). Gobierno del Estado hace más eficiente el Control Patrimonial. https://diariodecolima.com/noticias/ detalle/2019-02-20-gobierno-del-estado-hacems-eficiente-el-control-patrimonial

Cabrera, C. (2018). El control interno patrimonial y su incidencia en la gestión de los bienes muebles de la Municipalidad Provincial de Rioja - 2018 (Tesis para obtener el Título profesional de Contador Público, Universidad Cesar Vallejo). Repositorio Universidad Cesar Vallejo. https://repositorio.ucv.edu.pe/ bitstream/handle/20.500.12692/43006/Cabrera AC.pdf? sequence $=1$ \&isAllowed $=y$

Cruz, S. (2019). Control Interno del inventario en Sistema Educativo de Misión Ecuatoriana del Sur (Tesis para optar por el Titulo de Contador Público, Universidad de Guayaquil). Repositorio institucional de la Universidad de Guayaquil. http://repositorio.ug.edu.ec/bitstream/ redug/46407/ / Tesis $\% 20 \mathrm{~S}$ a mantha $\% 20$ Cruz\%20-\%20Control\%20interno\%20del\%20 inventario $\% 20 \mathrm{en} \% 20$ Sistema $\% 20$ Educativo $\% 20$ de\%20Mision\%20Ecuatoriana\%20del\%20Sur. pdf

Cuevas, L. (2018). Control interno del sector público. Comisión de Contabilidad y

Auditoría Gubernamental del Colegio de Contadores Públicos de México. Recuperado de: https://www.ccpm.org.mx/avisos/2018-2020/ control.interno.cofi.pdf

Estupiñan G. (2012). "Control interno y fraudes". Bogotá - Colombia, eco ediciones. 2006 https://www.ecoeediciones.com/wp-content/ uploads/2015/07/Control-interno-y-fraudes3ra-Edicio\%CC\%81n.pdf

Fonseca, O. (2011). Sistemas de Control Interno para Organizaciones. Instituto de Investigación de Accountability y Control - IICO.

García, B. y Nazareno, E. (2018). El control interno y su incidencia en la gestión de inventarios de la distribuidora JALDAB S.A [Proyecto de investigación para la obtención del título de 
Ingeniero Comercial, Universidad de Guayaquil]. Repositorio institucional de la Universidad de Guayaquil. http://repositorio.ug.edu.ec/ bitstream/redug/28045/1/Garc\%c3\%ada\%20 Salas_Nazareno\%20Bonaga.pdf

Gonzales, M. y Huamán, R. (2018). Diseño de un sistema de control patrimonial para disminuir los faltantes de bienes en la municipalidad distrital de Tumàn - 2012 [Tesis para optar título profesional de Contador Público, Universidad Señor de Sipan]. Repositorio institucional Universidad Señor de Sipán. https://repositorio. uss.edu.pe/handle/20.500.12802/4487

Gestión, D. (13 de agosto 2018). El 93\% de municipios provinciales ya cuenta con Órgano de Control de la Contraloría. https://gestion. $\mathrm{pe} / \mathrm{peru} /$ politica/93-municipios-provincialescuenta-organo-control-contraloria-241365noticia/?ref=gesr

Idrovo, E. y Córdova, M. (2018). Manual de control interno de inventarios aplicado para la hacienda bananera San Luis (Tesis para optar por el título de contador Público autorizado, Universidad de Guayaquil). Repositorio Institucional de Universidad de GuayaquilEcuador. http://repositorio.ug.edu.ec/handle/ redug/37568

Kirschenbaum, P \& Mangulan J; Enterprise Risk Managenent:; PriceWater HouseCoopers; Estados Unidos 2004. https://www.redalyc.org/ pdf/416/41648308010.pdf

Lozano, R.(2019). Análisis del control interno enla empresa la feria del constructor S.A.C. Ferreñafe, 2018 [Tesis de grado académico profesional de Bachiller en Contabilidad, Universidad Señor de Sipán]. Repositorio Institucional de Universidad Señor de Sipan. https://repositorio.uss.edu. pe/bitstream/handle/20.500.12802/6408/ Lozano\%20Bautista\%20Rosa\%20Yaneth. pdf? sequence $=1$ \&isAllowed $=y$

Mantilla, S. (2005). Control Interno: Un Mundo globalizado. Colombia: Editorial McGrawHill. https://www.ecoeediciones.com/wp-content/ uploads/2018/04/Auditori\%CC\%81a-delControl-Interno-4ed.pdf

Niño R. (2017). "Propuesta de implementación de control interno para mejorar la gestión de inventarios de la empresa de confecciones Ravsa Sport de la ciudad de Lambayeque - 2016.” (Tesis para optar por el título de contador Público autorizado, Universidad de Lambayeque). Repositorio Institucional de Universidad de Lambayeque. http://repositorio.udl.edu.pe/ handle/UDL/96

Organismo Supervisor de Contrataciones con el estado - OSCE (2019). Plan anual de contrataciones del año 2019. Lima, Perú.

https://cdn.www.gob.pe/uploads/document/ file/562579/Plan_Anual_de_Contrataciones_ del_a\%C3\%B1o_2019.pdf

Peralta, M. y Villavicencio, G. (2018). Evaluación del sistema de control interno de la empresa DINGROUP CÍA. LTDA. Y propuesta de mejora (Tesis para la obtención del Título Profesional de Contador Público, Universidad de Cuenca). Repositorio Institucional de Universidad de Cuenca. http://dspace.ucuenca.edu.ec/ bitstream/123456789/30111/1/Trabajo\%20 de\%20titulaci\%c3\%b3n.pdf

Ramón, C. (2019). Propuesta de un sistema de control de activos fijos para la Fiscalía Provincial de Loja para el año 2018 (Trabajo de Titulación previo a la obtención del título de Contador Público auditor, Universidad de Cuenca - Ecuador). Repositorio Institucional de Universidad de Cuenca.http://dspace. ucuenca.edu.ec/bitstream/123456789/31961/1/ Trabajo\%20de\%20titulacion.pdf

Sánchez, L. (2017). El control patrimonial en la administración de bienes muebles de la Municipalidad Provincial de Huánuco, 2017 [Tesis de Pregrado, Universidad de Huánuco]. Repositorio Institucional de la Universidad de Huánuco. http://repositorio.udh.edu.pe/ 